\title{
Bestimmung des freien Thyroxingehalts im Serum mittels Gelfiltration
}

\author{
Von G. Gyertyánfy, J. FöLdes, E. Gesztesi und I. TAkÁcs
}

Aus der I. Medizinischen Universitätsklinik (Direktor: Prof. Dr. I. Magyar) Budapest, Ungarn

(Eingegangen am 25. August 1967)

\begin{abstract}
Die Auswirkungen der Granulagröße und der Länge der Gelfiltrationssäule, des pH-Wertes der eluierenden Pufferlösung und der Verdünnung des Ausgangsserums bei der prozentualen Bestimmung des sog. „freien“ Thyroxins wurden untersucht, ebenso die Wirkung der Inkubationszeit und der Konzentration des inaktiven Thyroxins. Mit Hilfe von $\mathrm{Na}^{125} \mathrm{~J}$ und ${ }^{131} \mathrm{~J}$-Albumin wurde die Zuverlässigkeit der Methode kontrolliert. Den Ergebnissen zufolge erscheint die Gelfiltrationsmethode als geeignet zur prozentụalen Bestimmung des sog. „freien“ Thyroxins im Serum. Als Vorteil des Verfahrens wäre zu erwähnen, daß es von den Fehlern des HaMolsky-Verfahrens unabhängig ist.

The influence of the following factors on the percentage determination of so-called "free" thyroxin was studied: length of the column and particle size of the gel, $\mathrm{pH}$ of the eluant buffer and dilution of the starting serum, time of incubation and concentration of inactive thyroxin. The reliability of the method was investigated with the aid of $\mathrm{Na}^{125} \mathrm{I}$ and ${ }^{131} \mathrm{I}$-albumin. The results show that the gel filtration method is suitable for the percentage determination of the "free thyroxin" in serum. This method has the advantage that it is not subject to the errors of the HaMOLSKY method.
\end{abstract}

Im Serum zirkulieren die Schilddrüsenhormone an Eiweißstoffe gebunden und bleiben nur in geringer Menge „frei““. Nach gewissen Angaben (1) hängt der Stoffwechselzustand des Patienten eng mit der freien Hormonmenge zusammen. Sofern die Bildung der das Schilddrüsenhormon bindenden Eiweißstoffe ein normales Ausmaß zeigt, stellt das verbleibende („Reserve-“) Thyroxinbindungsvermögen der Serumeiweißstoffe einen guten Indikator des Funktionszustandes der Schilddrüse dar. So sind die Serumeiweißstoffe bei Hyperthyreose, bei welcher eine große Menge Schilddrüsenhormon entsteht, mit diesem verhältnismäßig gesättigt. Von exogen dem Serum zugegebenem markiertem Schilddrüsenhormon vermögen sie somit nur wenig aufzunehmen, so daß eine große Hormonmenge „frei“" bleibt. Das Gegenteil ist bei Hypothyreose zu beobachten.

Dem vorstehend skizzierten Prinzip entsprechend hat HAMOLSKY sein in-vitro-Verfahren mit markiertem Trijodthyronin (2) ausgearbeitet. Außer markiertem Trijodthyronin hat man auch Thyroxin zur Untersuchung des Funktionszustandes der Schilddrüse verwendet $(3,4)$.

Die Ergebnisse dieser Methoden werden von zahlreichen Faktoren (5-8) beeinflußt:

a) Von der $\mathrm{pH}$-Wertveränderung des Serums $\left(\mathrm{CO}_{2}-\right.$ Spannung),

b) von der Menge und Temperatur der Waschflüssigkeit sowie der Anzahl der Waschungen,

c) von der Hämolyse der Erythrocyten,

d) vom Hämatokritwert,

e) vom freien anorganischen Jodgehalt des zur invitro-Probe verwendeten markierten Hormons usw.

Zahlreiche Versuche wurden zur Elimination der obigen Faktoren unternommen (9-12).

In den letzten Jahren gewinnen mehr und mehr die auf Gelfiltration beruhenden Verfahren die Oberhand. Mehrere Autoren fanden, diese Methode eigne sich zur Trennung und Bestimmung von markiertem freiem
Thyroxin und Trijodthyronin (13-17). In vorliegender Mitteilung wollen wir über unsere diesbezüglichen Beobachtungen berichten.

Bei unseren Versuchen gingen wir von den Arbeiten von SCRIBa $(16,17)$ Hocman (14) und Clark (15) aus. Wir untersuchten, inwieweit die ermittelten Resultate vom Granulamaß des Dextran-Gels, von der Länge der Säule, vom pH-Wert des eluierenden Puffers, den Verdünnungsverhältnissen, der Inkubationszeit und der Quantität des zugegebenen inaktiven Thyroxins beeinflußt werden. Zugleich kontrollierten wir die Zuverläsșigkeit der Methode mit Hilfe von $\mathrm{Na}^{125} \mathrm{~J}$ und ${ }^{131} \mathrm{~J}$-Albumin. Hierbei wurde untersucht, ob das markierte anorganische Jodid und markiertes Eiweiß das Untersuchungsergebnis beeinflussen. Bei den Versuchen verwendeten wir markiertes Thyroxin, weil Thyroxin stets im Serum anwesend ist, Trijodthyronin dagegen fehlen kann. Von Thyroxin stammen mehr als $90 \%$ des eiweißgebundenen Jods. Überdies bindet sich Thyroxin in gewissem Maße stärker an die Serumeiweißstoffe als Trijodthyronin, so $\mathrm{da} ß$ wir die Untersuchung mit Thyroxin als physiologischer ansehen.

\section{Methodik und Ergebnisse}

Prinzip

Wird Serum mit einer Lösung inkubiert, die markiertes Thyroxin enthält, so bindet sich ein beträchtlicher Teil davon an die Serumeiweißstoffe $\left.\left(\mathrm{PB}^{125} \mathrm{Tx}\right)^{1}\right)$ und nur wenig bleibt „frei“. Wahrșcheinlich kommt nach dem Massenwirkungsgesetz ein dynamischer Gleichgewichtszustand zwischen dem „freien“" Thyroxin, dem eiweißgebundenen Thyroxin und den entspreçhenden Bindungseiweißstoffen zustande. Wird dieses Serum auf die Sephadex-Säule übertragen, so geht mit der eluierenden Pufferlösung dem gröBeren Molekulargewicht entsprechend zuerst die Elution des eiweißgebundenen Thyroxins vor sich, der in den weiteren Fraktionen das Jodid mit niedrigem Molekulargewicht nachfolgt. Dextran-Gel adsorbiert auch das von den Serumeiweißstoffen nicht gebundene "freie" Thyroxin. Dieses adsorbierte markierte „freie“ Thyroxin eluieren wir mit dem Serum eines gesunden Menschen von der Säule. Das Serum hat ein größeres Thyroxin-Bindungs-'

1) Abkürzungen: $\mathrm{PB}^{125} \mathrm{Tx}=$ Protein-gebundenes ${ }^{125} \mathrm{~J}$-Thyroxin, ${ }^{125} \mathrm{~J}-\mathrm{T}_{\mathrm{f}}=$ freies ${ }^{125} \mathrm{~J}$-Thyroxin, $\mathrm{Tx}=$ freies Thyroxin. 
vermögen als Dextran-Gel und ist daher imstande, letzteres von der Säule abzulösen. In den von der Säule abgelösten Fraktionen sind demnach drei radioaktive Spitzen anzutreffen, von denen die ersten beiden durch Elution mit der Pufferlösung gewonnen werden. Die erste - größte - Spitze entspricht dem $\mathrm{PB}^{125} \mathrm{Tx}$-, die zweite - kleinere - Spitze dem ${ }^{125} \mathrm{~J}$-Gehalt. Die dritte, durch Elution mit Serum gewonnene Spitze entspricht dem sog. „freien“ Thyroxin $\left({ }^{125} \mathrm{~J}-\mathrm{Tx}_{\mathbf{f}}\right)$.

\section{Reagenzien}

${ }^{125} \mathrm{~J}$-Thyroxin (Radiochemical Center, Amersham) $\mathrm{Na}^{125} \mathrm{~J}$ und ${ }^{131} \mathrm{~J}$-Albumin (Isotopen-Institut der Ungarischen Akademie der Wissenschaften).

Dextran-Gel Sephadex G-25, „coarse“ (Granulagröße 100

bis $300 \mu \mathrm{m}$ ) und ,fine“ (Granulagröße 20-80 $\mu \mathrm{m}$ )

(Pharmacia, Uppsala)

$0,01 \mathrm{M}$ Phosphatpuffer, $\mathrm{pH}=7,4$

$1 / 15 \mathrm{M}$ Phosphatpuffer, $\mathrm{pH}=9,5$

Inaktives DL-Thyroxin (Fluka)

Humanserum (Staatl. Blutversorgungsdienst).

Die Gelfiltrationssäulen wurden so hergestellt, daß wir DextranGel („fine" oder „,coarse") mit Phosphatpuffer $\mathrm{pH}=7,4$ oder $\mathrm{pH}=9,5$ drei Tage quellen ließen und dann 15, 23 bzw. $30 \mathrm{~cm}$ lange Säulen mit $1 \mathrm{~cm}$ Durchmesser bildeten.

\section{Untersuchung der Länge der Gelfiltrationssäule}

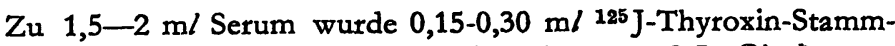
lösung (etwa 0,1-0,2 $\mu \mathrm{g} / \mathrm{ml}$, spez. Aktivität etwa $2-5 \mu \mathrm{C} / \mathrm{ml}$ ) gegeben, so daß die Konzentration an markiertem Thyroxin $1 \mu \mathrm{g} / 100 \mathrm{~m} l$ betrug. Die Aktivität der Proben entsprach jeweils $0,2-0,5 \mu \mathrm{C}$. Mit 0,01M Phosphatpuffer wurde auf das doppelte Volumen ergänzt. Ein $\mathrm{ml}$ dieses Substrates wird nach 1stdg. Inkubation bei $37^{\circ}$ auf die verschiedenen Sephadex-Säulen übertragen, während $1 \mathrm{~m} l$ als Standard dient. Die Elution erfolgt mit 0,01M Phosphatpuffer und Humanserum, wobei 15 Stück $1 \mathrm{~m} /$-Fraktionen gesammelt werden. Die Aktivität der Fraktionen wird im Szintillationskristall gemessen.

Benutzt man eine $15 \mathrm{~cm}$ lange "fine" - oder "coarse“Säule, so sind die Resultate nicht adäquat, die Rückgewinnung liegt zwischen 50 und $80 \%$. Eine befriedigende Rückgewinnung ist nur mit der $23 \mathrm{~cm}$ langen „fine“- und der $30 \mathrm{~cm}$ langen „coarse“-Säule zu erreichen.

Die Meßergebnisse veranschaulichen der obere Teil von Tabelle 1 und Abbildung 1. Wie ersichtlich, ist $\mathrm{PB}^{125} \mathrm{Tx}$ in den Fraktionen 4-8, das anorganische Jodid in den Fraktionen 10-14 und das sog. "freie" markierte Thyroxin in den Fraktionen 19-22 anzutreffen. Innerhalb jeder Versuchsreihe (s. Nr.) geben wir den Durchschnitt von zwei Experimenten an.

\section{Die Auswirkung des Granulamaßes auf die "freie" markierte Thyroxinmenge}

Die Versuche wurden an der $23 \mathrm{~cm}$ langen ,fine“- und $30 \mathrm{~cm}$ langen "coarse"-Säule vorgenommen. Die Vorbereitung des Serums, die Ablösung und Messung geschahen nach dem im vorigen Punkt beschriebenen Verfahren.

Die Resultate sind im mittleren Teil von Tabelle $1 \mathrm{zu}-$ sammengefaßt. In den Tabellenangaben betrachten wir die an der "fine"-Säule gewonnenen Werte als Ausgangsgrundlage $(100 \%)$ und beziehen auf diese die an der „coarse"-Säule gewonnenen Werte (Spalte 8), weil die Differenzen so deutlicher zutage treten. Bei Benutzung der "coarse"-Säule ergibt sich ein wesentlich höherer $\mathrm{PB}^{125} \mathrm{Tx}$-Gehalt als an der „fine"-Säule; das anorganische Jodid zeigt einen um durchschnittlich $20 \%$, das „freie“ Thyroxin einen um $30 \%$ niedrigeren Wert.

Abb. 1

Elutionsdiagramm der Gelfiltration von freiem Thyroxin. $\mathrm{Zu}$ Beginn wurden PBI und Jrdid (J) mit Puffer, dann freies Thyroxin (Tx) mit Serum eluiert

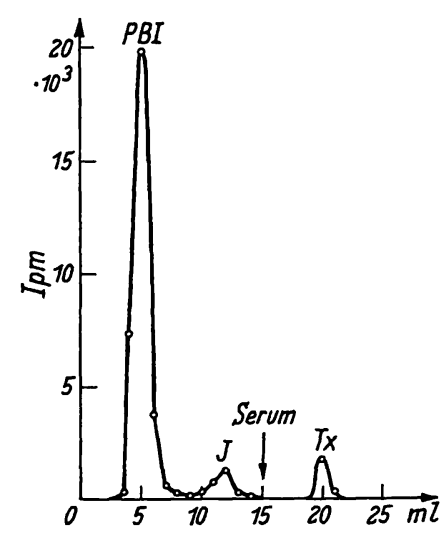

Tab. 1

Untersuchung der Gelfiltrationsergebnisse, Granulamaße und des pH-Einflusses

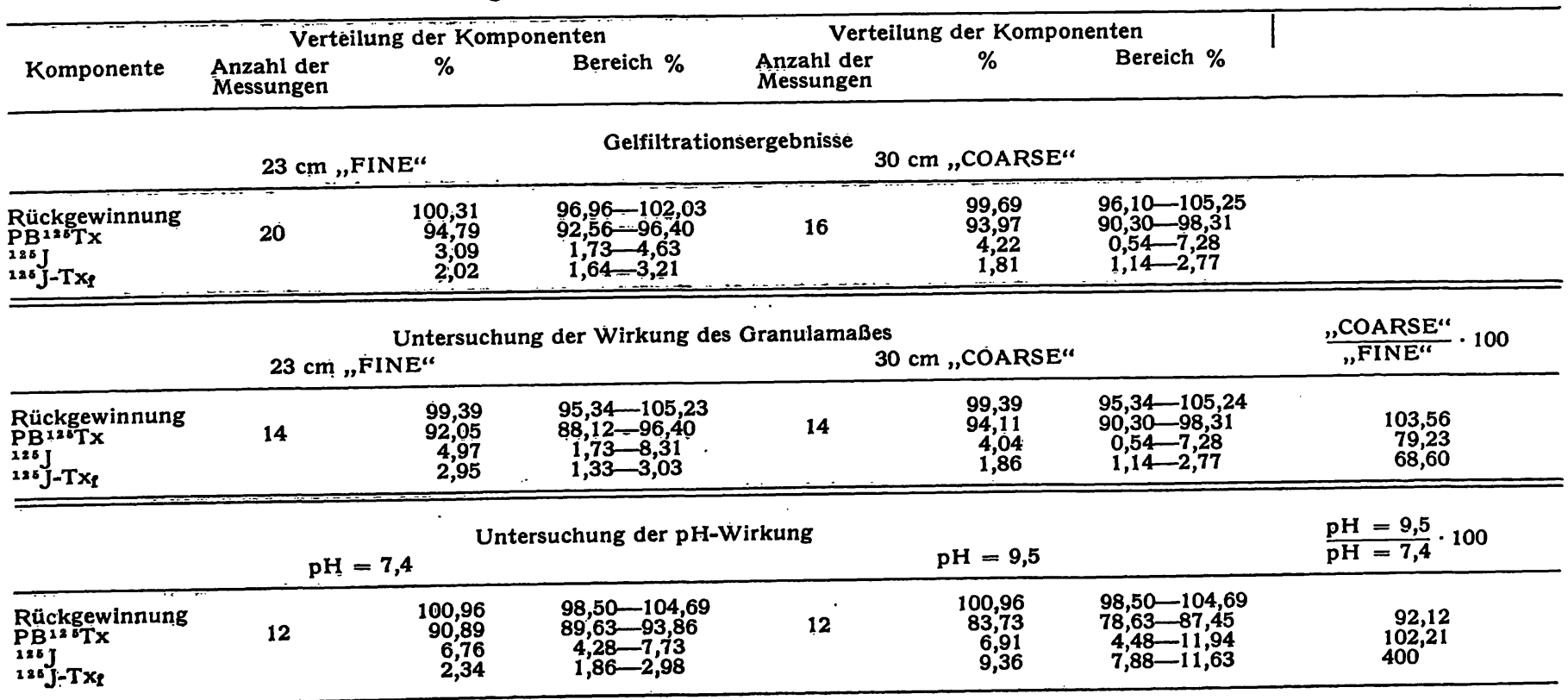


Tab. 2

Wanderungsresultate des ohne und mit Puffer inkubierten Serums

\begin{tabular}{|c|c|c|c|c|c|c|c|}
\hline \multirow[b]{2}{*}{ Komponente } & \multicolumn{3}{|c|}{ Verteilung der Komponenten } & \multicolumn{3}{|c|}{ Verteilung der Komponenten } & \multirow[b]{2}{*}{$\frac{\text { Mit Puffer } \cdot 100}{\text { Ohne Puffer }}$} \\
\hline & $\begin{array}{l}\text { Anzahl der } \\
\text { Messungen }\end{array}$ & $\%$ & Bereich \% & $\begin{array}{l}\text { Anzahl der } \\
\text { Messungen }\end{array}$ & $\%$ & Bereich \% & \\
\hline \multicolumn{8}{|c|}{ Wanderung des mit und ohne Puffer inkubierten Serums } \\
\hline & \multicolumn{2}{|c|}{ Ohne Puffer inkubiert } & \multicolumn{4}{|c|}{ Mit Puffer inkubiert } & \\
\hline \multirow[t]{3}{*}{$\begin{array}{l}\text { Rückgewinnung } \\
\text { PB } 125 \mathrm{Tx} \\
126 \mathrm{~J} \\
128 \mathrm{~J}-\mathrm{Tx}_{\mathrm{P}} \\
\end{array}$} & 8 & $\begin{array}{r}101,35 \\
95,04 \\
3,71 \\
1,25\end{array}$ & $\begin{array}{c}96,14-105,95 \\
94,12-96,36 \\
2,63-4,63 \\
1,05-1,37\end{array}$ & 8 & $\begin{array}{r}100,34 \\
94,52 \\
3,39 \\
2,09\end{array}$ & $\begin{array}{c}96,96-104,14 \\
93,82-96,13 \\
2,01-4,13 \\
1,86-2,43\end{array}$ & $\begin{array}{r}99,45 \\
90,80 \\
167,92\end{array}$ \\
\hline & \multirow{2}{*}{\multicolumn{4}{|c|}{ Ohne Puffer inkubiert }} & & & 1. Wanderung $\cdot 100$ \\
\hline & & & & & 2. Wander & & 2. Wanderung \\
\hline \multirow[t]{3}{*}{$\begin{array}{l}\text { Rückgewinnung } \\
\mathrm{PB}_{125}^{125 \mathrm{TX}} \\
126 \mathrm{~J} \\
125 \mathrm{~J}-\mathrm{TX}_{\mathrm{P}} \\
\end{array}$} & 8 & $\begin{array}{r}101,35 \\
95,04 \\
3,71 \\
1,25\end{array}$ & $\begin{array}{c}96,14-105,95 \\
94,12-96,32 \\
2,63-4,63 \\
1,05-1,37 \\
\end{array}$ & 8 & $\begin{array}{r}100,34 \\
96,80 \\
3,70\end{array}$ & $\begin{array}{c}96,96-104,14 \\
95,13-97,80 \\
2,20-4,87 \\
\end{array}$ & $\begin{array}{l}101,32 \\
292,38 \\
\end{array}$ \\
\hline & \multirow{2}{*}{\multicolumn{4}{|c|}{ Mit Puffer inkubiert }} & & & 1. Wanderung $\cdot 100$ \\
\hline & & & & & 2. Wander & & 2. Wanderung \\
\hline $\begin{array}{l}\mathrm{PB}_{125}^{125} \mathrm{Tx} \\
125 \mathrm{~J}-\mathrm{TxP}\end{array}$ & 16 & $\begin{array}{r}93,47 \\
4,30 \\
2,23\end{array}$ & $\begin{array}{c}90,30-94,47 \\
2,01-7,28 \\
1,59-3,21\end{array}$ & 16 & $\frac{93,66}{6,71}$ & $\begin{array}{r}88,76-95,54 \\
4,26-11,24\end{array}$ & $\frac{100,23}{313,74}$ \\
\hline
\end{tabular}

\section{Untersucbung der $p H$-Wirkung}

Wir gehen von $1,5 \mathrm{~m} /$ Serum aus, das nach Punkt $1 \mathrm{mit} 0,01 \mathrm{M}$ oder 1/15M Phosphatpuffer vorbereitet, eluiert und gemessen wird.

Die Ergebnisse veranschaulicht der untere Teil von Tabelle 1. Die mit dem Puffer $\mathrm{pH}=7,4$ gewonnenen Werte erachten wir als $100 \%$ und setzen zu diesen die mit dem Puffer $\mathrm{pH}=9,5$ erhaltenen Resultate ins Verhältnis. Bei Anwendung von Puffer $\mathrm{pH}=9,5$ ist der Wert von $\mathrm{PB}^{125} \mathrm{Tx}$ stets niedriger als der mit dem Puffer $0,01 \mathrm{M} \mathrm{pH}=7,4$ gewonnene. Die anorganische Jodidmenge schwankt. Die „freie“ Thyroxinmenge ergibt mit dem Puffer $\mathrm{pH}=9,5$ stets einen 3-4mal größeren Wert als mit dem Puffer $\mathrm{pH}=7,4$.

\section{Untersuchung der Verdünnungswirkung}

Wir untersuchten das Verhalten des „freien“ Thyroxins bei Inkubation ohne und mit Puffer, weiterhin, was geschieht, wenn die $\mathrm{PB}^{125} \mathrm{Tx}$-Fraktion einer weiteren Gelfiltration unterworfen wird.

a) Zum Vergleich der Wanderung des ohne und mit Puffer inkubierten Serums gingen wir wie folgt vor: $\mathrm{Zu} 3 \mathrm{~m} l$ Serum wurde
$0,15-0,30 \mathrm{ml}{ }^{125} \mathrm{~J}$-Thyroxin-Stammlösung (s. o.) gegeben, so $\mathrm{da} \beta$ die Konzentration an markierten Thyroxin $1 \mu \mathrm{g} / 100 \mathrm{ml}$ betrug. Die Aktivität der Probe entsprach 0,2-0,4 $\mu \mathrm{C}$. Dieses Substrat wurde (ohne Puffer) 1 Std. bis $37^{\circ}$ inkubiert. Als Standard diente $1 \mathrm{~m} l$ dieses Ansatzes, ebenso wurde $1 \mathrm{~m} l$ davon auf die $23 \mathrm{~cm}$ lange „fine“-Säule übertragen, wonach wir wie unter 1. verfuhren. Zur gleichen Zeit wurden von demselben Serum $1,5 \mathrm{~m} l$ nach dem unter 1 . beschhriebenen Verfahren (d. $h$. mit Puffer) vorbereitet, aufgetragen, eluiert und gemessen.

Die Ergebnisse sind in Tabelle 2 zusammengefaßt. Wir sehen die bei der Wanderung des ohne Puffer inkubierten Serums gewonnenen Werte als $100 \%$ an und setzen die Werte des mit Puffer inkubierten Serums hierzu ins Verhältnis. Dabei zeigt der $\mathrm{PB}^{125} \mathrm{Tx}$-Gehalt keine wesentliche Veränderung, die anorganische Jodidmenge ist durchschnittlich um $9 \%$ verringert, die „freie" Thyroxinmenge hingegen wesentlich vermehrt. b) Vorbereitung der Serumproben und weiteres Vorgehen wie nach Punkt 4a) ohne Puffer.

Meßergebnisse Tabelle 2 Mitte. Betrachten wir die anläßlich der ersten Wanderung erhaltenen Werte als

Tab. 3

Wirkung des Grades der Serumverdünnung und der Konzentration des inaktiven Thyroxins auf die Verteilung der Komponenten

\begin{tabular}{|c|c|c|c|c|c|c|c|c|c|c|}
\hline \multirow{2}{*}{$\begin{array}{l}\text { Nr. } \\
1 .\end{array}$} & \multirow{2}{*}{$\begin{array}{l}\text { Komponente } \\
{ }_{125}^{P_{125} \mathrm{Jx}} \\
{ }^{126} \mathrm{~J}-\mathrm{TX}_{\ell}\end{array}$} & \multirow{2}{*}{$\begin{array}{c}0 \\
83,93 \\
14,76 \\
1,31\end{array}$} & \multicolumn{3}{|c|}{ 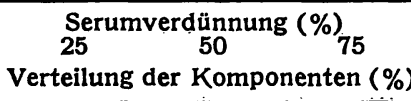 } & \multirow{2}{*}{$\begin{array}{r}90 \\
71,69 \\
18,80 \\
9,51\end{array}$} & \multicolumn{4}{|c|}{ 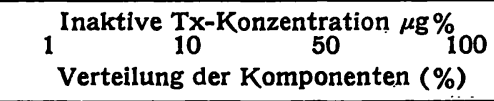 } \\
\hline & & & $\begin{array}{r}81,98 \\
16,22 \\
1,79\end{array}$ & $\begin{array}{r}80,52 \\
17,07 \\
2,42\end{array}$ & $\begin{array}{r}80,21 \\
15,31 \\
3,66\end{array}$ & & $\begin{array}{r}90,98 \\
6,79 \\
2,23\end{array}$ & $\begin{array}{r}88,70 \\
7,23 \\
4,06\end{array}$ & $\begin{array}{r}89,91 \\
6,50 \\
3,59\end{array}$ & $\begin{array}{r}86,92 \\
6,69 \\
6,09\end{array}$ \\
\hline 2. & $\begin{array}{l}\mathrm{PB}_{125}^{125} \mathrm{Tx} \\
125 \mathrm{~J}-\mathrm{TX}_{\mathrm{P}}\end{array}$ & $\begin{array}{r}94,39 \\
4,46 \\
1,15\end{array}$ & $\begin{array}{r}93,70 \\
5,06 \\
1,24\end{array}$ & $\begin{array}{r}94,53 \\
3,93 \\
1,54\end{array}$ & $\begin{array}{r}92,34 \\
4,28 \\
3,38\end{array}$ & $\begin{array}{r}86,66 \\
5,32 \\
8,03\end{array}$ & $\begin{array}{r}90,07 \\
7,42 \\
2,51\end{array}$ & $\begin{array}{r}88,17 \\
8,83 \\
2,99\end{array}$ & $\begin{array}{r}85,19 \\
9,44 \\
5,36\end{array}$ & $\begin{array}{r}86,13 \\
7,59 \\
6,28\end{array}$ \\
\hline 3. $\left.{ }^{1}\right)$ & $\begin{array}{l}\mathrm{PB}_{125}^{125 \mathrm{TX}} \\
126 \mathrm{~J}-\mathrm{TX}_{\mathrm{P}}\end{array}$ & $\begin{array}{r}96,17 \\
3,53 \\
0,48\end{array}$ & $\begin{array}{r}91,79 \\
7,45 \\
0,76\end{array}$ & $\begin{array}{r}94,53 \\
4,49 \\
0,98\end{array}$ & $\begin{array}{r}94,33 \\
3,93 \\
1,71\end{array}$ & $\begin{array}{r}89,20 \\
7,48 \\
3,31\end{array}$ & $\begin{array}{r}89,17 \\
8,51 \\
2,32\end{array}$ & $\begin{array}{r}87,50 \\
9,78 \\
2,72\end{array}$ & $\begin{array}{r}87,08 \\
9,46 \\
3,46\end{array}$ & $\begin{array}{r}86,24 \\
9,43 \\
4,38 \\
\end{array}$ \\
\hline \multirow[t]{3}{*}{ Durchschnitt } & $\begin{array}{l}\mathrm{PB}_{126 \mathrm{TX}} \\
126 \mathrm{~J} \\
126 \mathrm{~J}-\mathrm{Tx}_{\mathrm{l}}\end{array}$ & $\begin{array}{r}91,50 \\
7,58 \\
0,98\end{array}$ & $\begin{array}{r}89,16 \\
9,58 \\
1,26\end{array}$ & $\begin{array}{r}89,86 \\
8,50 \\
1,98\end{array}$ & $\begin{array}{r}88,96 \\
7,84 \\
3,26\end{array}$ & $\begin{array}{r}82,52 \\
10,53 \\
6,95\end{array}$ & $\begin{array}{r}90,07 \\
7,52 \\
2,53\end{array}$ & $\begin{array}{r}88,12 \\
8,61 \\
3,26\end{array}$ & $\begin{array}{r}87,39 \\
8,47 \\
4,10\end{array}$ & $\begin{array}{r}86,43 \\
7,90 \\
5,58\end{array}$ \\
\hline & \multicolumn{2}{|c|}{ Rückgewinnungsdurchschnitt } & \multicolumn{4}{|c|}{$99,01 \%$} & \multicolumn{4}{|c|}{$101,11 \%$} \\
\hline & \multicolumn{2}{|c|}{ Rückgewinnungsbereich } & \multicolumn{4}{|c|}{$96,75-102,33 \%$} & \multicolumn{4}{|c|}{$97,12-103,44 \%$} \\
\hline
\end{tabular}

2) Diese Messung wurde bei der Untersuchung des verdünnten Serums an einer $30 \mathrm{~cm}$ langen "coarse"-Säule vorgenommen. 


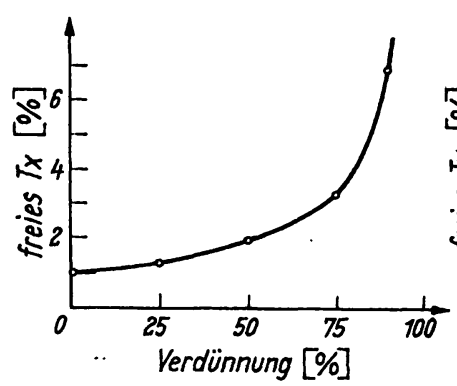

Abb. 2 den Gehalt an freiem Thyroxin
Wirkung der Serumverdünnung auf

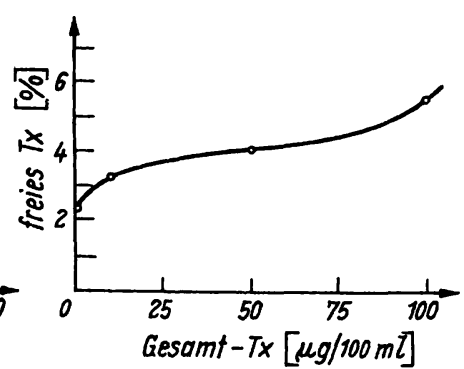

Abb. 3

Wirkung der ThyroxinkonzenThyroxin tration auf den Gehalt an freiem

$100 \%$, so sehen wir, daß die „freie" Thyroxinmenge bei der zweiten Wanderung beträchtlich zunimmt.

c) Vorbereitung des Serums wie bei 1, weiter wie bei $4 \mathrm{a}$.

d) Mit 0,01M Phosphatpuffer stellten wir eine 25, 50, 75 und 90 proz. Serum-Verdünnung her. $\mathrm{Zu} 3 \mathrm{ml}$ des so verdünnten Serums geben wir $0,15-0,30 \mathrm{~m} /{ }^{125} \mathrm{~J}$-Thyroxinlösung, (s. o.), so daß die Konzentration an markierten Thyroxin $1 \mu \mathrm{g} / 100 \mathrm{ml}$ betrug. Die Aktivität der Probe entsprach wieder 0,2-0,4 $\mu \mathrm{C}$. Außerdem stellen wir ebenso eine unverdünnte Serumprobe her. Im weiteren gehen wir wie unter Punkt 1. vor.

Die Meßergebnisse veranschaulichen die linke Seite von Tabelle 3 und Abbildung 2. Die „freie" Thyroxinmenge vermehrt sich mit steigender Verdünnung erst langsam, dann immer steiler. Die anorganische Jodidmenge nimmt erst bei 90proz. Verdünnung eindeutig $z u$, ähnlich zeigt die $\mathrm{PB}^{125} \mathrm{Tx}$-Menge erst bei dieser hochgradigen Verdünnung eine wesentliche Verminderung.

\section{Untersuchung des Effektes der Inkubationszeit}

Wir inkubieren 5 Proben, wie unter 1. vorbereitet, 15, 30, 60, 90 bzw. $120 \mathrm{Min}$. bei $37^{\circ}$, wonach Gelfiltration und Messung wie unter 1. erfolgten.
Die Meßergebnisse sind in Tabelle 4 angeführt. Nach den Tabellenangaben übt die Inkubationszeit keinen wesentlichen Einfluß auf die Resultate aus.

Tab. 4

Einfluß der Inkubationszeit

\begin{tabular}{|c|c|c|c|c|c|c|}
\hline \multicolumn{2}{|c|}{ Nr. Kompo- } & 15 & \multicolumn{3}{|c|}{ 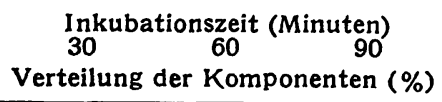 } & 120 \\
\hline 1 & $\begin{array}{l}\mathrm{PB}^{125} \mathrm{TX} \\
{ }^{128} \mathrm{~J} \\
{ }^{128} \mathrm{~J}-\mathrm{TXP}\end{array}$ & $\begin{array}{r}86,57 \\
11,23 \\
2,19\end{array}$ & $\begin{array}{r}86,31 \\
11,15 \\
2,54\end{array}$ & $\begin{array}{r}87,84 \\
10,05 \\
2,11\end{array}$ & $\begin{array}{r}87,38 \\
10,67 \\
1,95\end{array}$ & $\begin{array}{r}86,62 \\
10,72 \\
2,66\end{array}$ \\
\hline \multirow[t]{3}{*}{2} & $\begin{array}{l}\mathrm{PB}+25 \mathrm{TX} \\
{ }_{128} \mathrm{~J} \\
{ }_{128} \mathrm{~J}-\mathrm{TX}_{\mathrm{f}}\end{array}$ & $\begin{array}{r}93,46 \\
4,83 \\
1,71\end{array}$ & $\begin{array}{r}93,61 \\
4,53 \\
1,86\end{array}$ & $\begin{array}{r}93,06 \\
5,12 \\
1,82\end{array}$ & $\begin{array}{r}93,17 \\
5,02 \\
1,81\end{array}$ & $\begin{array}{r}93,12 \\
5,09 \\
1,79\end{array}$ \\
\hline & Rüc & gewinnt & Igsdurchsc & & $46 \%$ & \\
\hline & Rüc & gewinnt & gsbereich & 95,7 & $05,75 \%$ & \\
\hline
\end{tabular}

\section{Untersucbung des Effeletes der Thyroxinkonzentration}

Von der inaktiven Thyroxinlösung und vom Serum stellen wir Lösungen mit den Thyroxin-Konzentrationen 1, 10, 50,100 $\mu \mathrm{g} /$ $100 \mathrm{~m} l$ her, wonach die Vorbereitung, Inkubation, Gelfiltration und Messung wie bei 1. durchgeführt werden.

Meßergebnisse im rechten Teil von Tabelle 4 und in Abbildung 3. Die markierte „freie“ Thyroxinmenge steigt bei Vermehrung der inaktiven Thyroxinkonzentration erst langsamer, später etwas rascher an.

\section{Kontrolle der Zuverlässigkeit des Verfabrens mit $\mathrm{Na}^{125} \mathrm{~J}$}

$\mathrm{Zu} 1,5 \mathrm{ml}$ Serum wird $0,2-0,4 \mu \mathrm{C} \mathrm{Na}^{125} \mathrm{~J}$ aus einer Stammlösung (spez. Aktivität 2-4 $\mu \mathrm{C} / \mathrm{m} l$ ) zugegeben, und im weiteren gehen wir wie bei 1. vor.

Tab. 5

Kontrolle der Methode mit Hilfe von $\mathrm{Na}^{125} \mathrm{~J}$ und ${ }^{131} \mathrm{~J}$-Albumin

\begin{tabular}{|c|c|c|c|c|c|c|c|}
\hline \multirow{4}{*}{$\begin{array}{c}\text { Nr. } \\
1\end{array}$} & \multirow{4}{*}{$\begin{array}{l}\begin{array}{c}\text { Kompo- } \\
\text { nente }\end{array} \\
\begin{array}{l}\left.\mathrm{PBJ}^{1}\right) \\
\left.\mathrm{J}^{2}\right) \\
\left.\mathrm{T}_{\mathbf{f}^{2}}\right)\end{array}\end{array}$} & \multicolumn{3}{|c|}{$\begin{array}{l}\mathrm{Na}^{128} \mathrm{~J} \\
\text { Säule }\end{array}$} & \multicolumn{3}{|c|}{$\begin{array}{l}131 \mathrm{~J}-\text { Albumin } \\
\text { Säule }\end{array}$} \\
\hline & & $\begin{array}{l}15 \mathrm{~cm} \\
\text { "Coarse" }\end{array}$ & $\begin{array}{l}30 \mathrm{~cm} \\
\text {,Coarșe" }\end{array}$ & ,Fine & $\begin{array}{l}15 \mathrm{~cm} \\
\text { „Coarse“ }\end{array}$ & $\begin{array}{l}30 \mathrm{~cm} \\
\text { "Coarse" }\end{array}$ & $\begin{array}{l}23 \mathrm{~cm} \\
\text {,Fine }\end{array}$ \\
\hline & & \multicolumn{3}{|c|}{ Verteilung der Komponenten (\%) } & \multicolumn{3}{|c|}{ Verteilung der Komponenten (\%) } \\
\hline & & 100 & $\begin{array}{r}0,18 \\
99,82 \\
-\end{array}$ & $\begin{array}{r}0,24 \\
99,76 \\
-\end{array}$ & ${ }^{100}=$ & $\begin{array}{r}95,82 \\
4,18 \\
\end{array}$ & $\begin{array}{r}93,76 \\
6,24 \\
-\end{array}$ \\
\hline 2 & $\begin{array}{l}\mathrm{PBJ} \\
\mathbf{T} \mathrm{x}_{\mathbf{f}}\end{array}$ & $\begin{array}{r}0,28 \\
99,72 \\
=\end{array}$ & $\begin{array}{r}0,31 \\
99,69 \\
-\end{array}$ & $\begin{array}{r}0,37 \\
99,63 \\
-\end{array}$ & $\begin{array}{r}97,88 \\
2,12 \\
\end{array}$ & $\begin{array}{r}95,44 \\
4,56 \\
\end{array}$ & $\begin{array}{r}94,52 \\
5,48 \\
-\end{array}$ \\
\hline 3 & $\begin{array}{l}\mathbf{P B J} \\
\mathbf{T}_{\mathbf{P}}\end{array}$ & $\begin{array}{r}0,47 \\
99,53 \\
-\end{array}$ & $\begin{array}{r}3,50 \\
96,50 \\
-\end{array}$ & $\begin{array}{r}2,71 \\
97,29 \\
-\end{array}$ & $\begin{array}{r}95,92 \\
4,08 \\
=\end{array}$ & $\begin{array}{r}96,30 \\
3,70 \\
\end{array}$ & $\begin{array}{r}94,86 \\
5,14 \\
-\end{array}$ \\
\hline 4 & $\begin{array}{l}\mathbf{P B J} \\
\mathbf{J} \\
\mathbf{T} \mathbf{x}_{\mathbf{L}}\end{array}$ & 100 & $\begin{array}{r}3,29 \\
96,71 \\
\end{array}$ & $\begin{array}{r}4,04 \\
95,96 \\
\end{array}$ & $\begin{array}{r}95,67 \\
4,33 \\
-\end{array}$ & $\begin{array}{r}96,46 \\
3,54 \\
\end{array}$ & $\begin{array}{r}94,24 \\
5,76 \\
\end{array}$ \\
\hline 5 & $\begin{array}{l}\mathbf{P B J} \\
\mathbf{J}_{\mathbf{T}} \mathbf{I}\end{array}$ & $\begin{array}{r}2,37 \\
97,63 \\
=\end{array}$ & $\begin{array}{r}1,39 \\
98,61 \\
-\end{array}$ & $\begin{array}{r}0,44 \\
99,56\end{array}$ & $\begin{array}{r}96,43 \\
3,57 \\
-\end{array}$ & $\begin{array}{r}96,51 \\
3,49 \\
-\end{array}$ & $\begin{array}{r}95,10 \\
4,90 \\
\end{array}$ \\
\hline 6 & $\begin{array}{l}\text { PBJ } \\
\mathbf{J}_{\mathbf{T}} \mathbf{P}\end{array}$ & & & & $\begin{array}{r}97,04 \\
2,96 \\
\end{array}$ & $\begin{array}{r}96,85 \\
3,15 \\
-\end{array}$ & $\begin{array}{r}95,86 \\
4,14 \\
\end{array}$ \\
\hline $\begin{array}{l}\text { Durch- } \\
\text { schnitt }\end{array}$ & $\begin{array}{l}\text { PBJ } \\
\underset{\mathbf{T}}{\mathbf{T}} \mathbf{x}_{\mathfrak{l}}\end{array}$ & $\begin{array}{r}0,62 \\
99,37 \\
-\end{array}$ & $\begin{array}{r}1,73 \\
98,27 \\
-\end{array}$ & $\begin{array}{r}1,56 \\
98,45 \\
-\end{array}$ & $\begin{array}{r}97,16 \\
2,85 \\
-\end{array}$ & $\begin{array}{r}96,23 \\
3,77 \\
-\end{array}$ & $\begin{array}{c}94,72 \\
5,28 \\
-\end{array}$ \\
\hline
\end{tabular}

1) Bei Zusatz von $\mathrm{Na}^{125} \mathrm{~J}$ als $\mathrm{PB}^{128} \mathrm{~J}$, von ${ }^{131} \mathrm{~J}$-Albumin als $\mathrm{PB}^{131} \mathrm{~J}$.

2) Bei Zusatz von $\mathrm{Na}^{125 \mathrm{~J}}$ als ${ }^{225} \mathrm{~J}$, von ${ }^{281} \mathrm{~J}-\mathrm{Albumin}^{2}$ als ${ }^{101 \mathrm{~J}}$. 
Die Resultate sind auf der linken Seite von Tabelle 5 und in Abbildung 4 dargestellt. Bei einer unwesentlichen $\mathrm{PB}^{125} \mathrm{~J}$-Menge machen die anorganisches Jodid enthaltenden Fraktionen $98-100 \%$ der Gesamtaktivität aus, während wir freies Thyroxin bei keinem einzigen Versuch nachzuweisen vermochten.

\section{Kontrolle des Verfabrens mit ${ }^{131} \mathrm{~J}$-Albumin}

$\mathrm{Zu} 1,5 \mathrm{~m} l$ Serum wird $0,2-0,4 \mu \mathrm{C}{ }^{131} \mathrm{~J}$-Albumin aus einer Stammlösung (spez. Aktivität $2-4 \mu \mathrm{C} / \mathrm{m} l$ ) zugesetzt und wie unter 7. verfahren.

Die Ergebnisse sind auf der rechten Seite von Tabelle 5 und Abbildung $5 \mathrm{zu}$ sehen. Bei einem 94-97proz. $\mathrm{PB}^{131} \mathrm{~J}$-Gehalt sind $2-5 \%$ anorganisches Jodid anwesend, während freies Thyroxin nicht aufgezeigt werden kann.

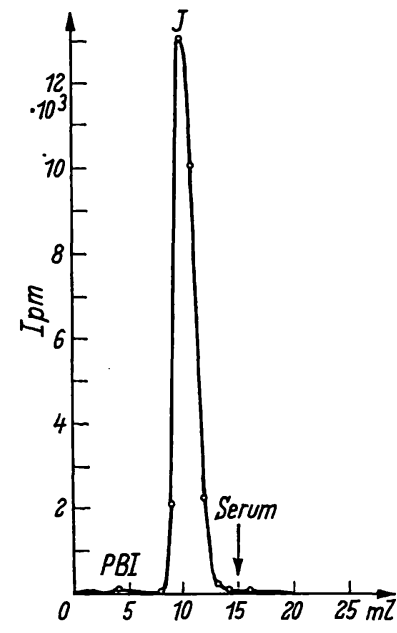

Abb. 4

Elutionsdiagramm der Gelfiltration von $\mathrm{Na}^{125} \mathrm{~J}$. S. a. Abb. 1

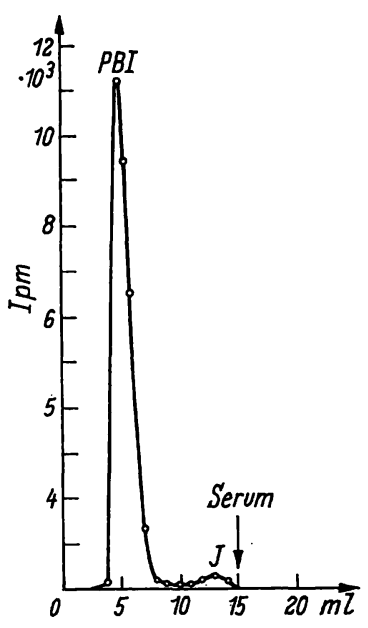

Abb. 5

Elutionsdiagramm der Gelfiltration von ${ }^{131} \mathrm{~J}$-Albumin s.a. Abb. 1

\section{Diskussion}

SCRIBA und Mitarbeiter (16, 17) haben Dextran-Gel Sephadex G-25 mit guten Ergebnissen zum Nachweis und zur Bestimmung des prozentualen Gehalts von sog. „freiem" Thyroxin im Serum angewendet. Nach unseren Befunden läßt sich die $23 \mathrm{~cm}$ lange ,fine"Säule mit einem Granulamaß von $20-80 \mu \mathrm{m}$ gut für diese Methode benutzen. Eine kürzere Säule können wir nicht empfehlen, weil das markierte Hormon nicht quantitativ zurückgewonnen werden kann. Bei Benutzung der $30 \mathrm{~cm}$ langen „coarse"-Säule mit einem Granulamaß von $100-300 \mu \mathrm{m}$ kann $z$ war das markierte Hormon quantitativ zurückgewonnen werden, doch werden die einzelnen Fraktionen nicht so scharf wie beim Gebrauch der "fine“-Säule voneinander getrennt. In der Praxis hat sich daher die $23 \mathrm{~cm}$ lange "fine“-Säule am besten bewährt.

Die verschiedenen Autoren wenden bei der Gelfiltration Puffer mit jeweils verschiedenem $\mathrm{pH}$-Wert an (13-17). Im Rahmen unserer Untersuchungen haben wir Phosphatpuffer $0,01 \mathrm{M} \mathrm{pH}=7,4$ und $1 / 15 \mathrm{M} \mathrm{pH}=9,5$ miteinander verglichen. Nach unseren Befunden ist bei einem höheren $\mathrm{pH}$-Wert die $\mathrm{PB}^{125} \mathrm{Tx}$ - und anorganische
Jodidmenge geringer, dagegen die freie Thyroxinmenge größer. Dies beruht vermutlich darauf, daß die Serumeiweißstoffe im alkalischeren Medium eine Schädigung erleiden und das Schilddrüsenhormon weniger $\mathrm{zu}$ binden vermögen $(18,19)$. Demnach empfiehlt sich die Anwendung von $0,01 \mathrm{M}$ Phosphatpuffer $\mathrm{pH}=7,4$.

Mit der Verdünnungsfrage müssen wir uns besonders befassen. CLARK und Mitarbeiter (15) haben das Serum im Verhältnis 1:50, SCRIBA und Mitarbeiter $(16,17)$ im Verhältnis 1:1 mit Puffer verdünnt. Wie das eingehende Studium der Frage ergibt, tritt, wenn man unverdünntes Serum untersucht, ein niedrigerer freier Thyroxin-Prozentwert zutage als beim verdünnten Serum, d. h. die freie Thyroxinmenge wird durch die Verdünnung eindeutig vermehrt. Dies ist darauf zurückzüuühren, daß mit dem Grad der Verdünnung die Menge der bindungsfähigen Stellen oder richtiger die Konzentration der bindungsfähigen Moleküle abnimmt. In Anbetracht dessen, daß die von SCRIBA und Mitarbeiter $(16,17)$ angewendete 50proz. Verdünnung (1:1) noch auf den flacheren Abschnitt der Kurve entfällt, ist diese Verdünnung noch akzeptabel.

Die einzelnen Autoren wenden auch eine unterschiedliche Inkubationszeit an. Deshalb untersuchten wir, ob eine Korrelation zwischen den Ergebnissen und der Inkubationszeit vorliegt. Nach unseren Angaben bindet sich das Thyroxin bereits innerhālb von 15 Min. maximal an die Serumeiweißstoffe. Eine Veräänderung der Inkubationszeit übt keinen Einfluß auf die Resultate aus. Wir empfehlen der Sicherheit halber eine 1stdg. Inkubationszeit.

Vermehrt man die Thyroxinmenge im Sinne des Massenwirkungsgesetzes, so wird das freie Bindungsvermögen der Eiweißstoffe geringer und vermehrt sich die freie Thyroxinmenge, was die Beobachtungen anderer Autoren $(15,16,20)$ bestätigt. Wird die Konzentration des inaktiven Thyroxins erhöht, so vermehrt sich die markierte freie Thyroxinmenge. Bei der Untersuchung benutzt man zweckmäßigerweise $1-2 \mu \mathrm{g}$ Thyroxin $/ 100 \mathrm{~m} l$ Serum.

Wir erachten es als nötig, die Zuverlässigkeit der Methode auch mit $\mathrm{Na}^{125} \mathrm{~J}$ und ${ }^{131} \mathrm{~J}$-Albumin zu kontrollieren. Nach unseren Ergebnissen läßt sich keines von beiden zusammen mit dem freien Thyroxin eluieren, so $\mathrm{da} \beta$ die prozentuale Bestimmung des freien Thytoxins durch ihre Anwesenheit nicht beeinflußt wird.

Die eigenen Beobachtungen zusammenfassend, dürfen wir feststellen, daß sich die auf der Gelfiltration beruhende Methode zur Bestimmung des sog. „freien“ Thyroxingehalts im Serum eignet. Zahlreiche Faktoren beeinflussen das Verfahren, weswegen konstante Standardbedingungen gewährleistet werden müsșen. Mit Hilfe der Gelfiltration werden die Fehlerquellen des Verfahrens von Hamolsky eliminiert.

Wir danken dem Isotopenlaboratorium des Zentralforschungsinstituts im Staatl. Bluttransfusionsdienst sowie Herrn Dr. G. GÁRDos für die freundliche Uberlassung der erforderlichen Serummenge. 


\title{
Literatur
}

1. Robins, J. und J. E. Rald, Recent Progr. Hormone Res., 13, 161 (1957). - 2. HAMolsky, M. W., M. Stein und A. S. Freedberg, J. Clin. Endocr. (Springfield) 17, 33 (1957). - 3. Ferrinr, O., G. L. Perroni, P. Biassini und M. Bestagno, Minerva Nucleare, 6, 49 (1962). - 4. Sirger, E. und A. SACrs, J. Clin. Endocr. (Springfield), 24, 313 (1964). - 5. AdaMs, R. A., N. SPECht und I. Woodward, J. Clin. Endoct. (Springfield) 20, 1366 (1960). - 6. Walfish, P. G., A. Britton, R. Volpe und C. Ezrin, Canad. Med. Ass. J., 84, 637 (1961). - 7. TAuxe, N. W., J. L. Becton und M. Y. Yamaguchr, Amer. J. Clin. Path. 39, 562 (1963). - 8. Woldring, M. G., A. BAKker und H. DoorenBos, Acta endocr. K'hrn 37, 607 (1961). - 9. SterLing, K. und M. Tabacrinik, J. Clin. Endocr. (Springfield) 21, 456 (1961). 10. McAdams, G. B. und R. F. Reinfrank, J. Nucl. Med. 5, 112
(1964). - 11. Lee, N. D., V. J. Pileggr und M. Segalove, Clin. Chem. (New York) 10, 136 (1964). - 12. F. Clark und D. B. HORN, J. Clin. Endocr. (Springfield) 26, 352 (1966). - 13. LEE, N. D., R. J. Henry und O. J. Golub, J. Clin. Endocr. (Springfield) 24, 486 (1964). - 14. Hocman, G., J. Chromatogr. (Amsterdam) 21, 413 (1966). - 15. ClaArk, F., G. Goldstein und K. R. Crispeli, J. Nucl. Med. 7, 464 (1966). - 16. SCribe, P. C., R. LaNdGRAF, H. G. HeINZE und K. SChWARZ, Klin. Wschr. 44, 69 (1966). 17. Scriba, P. C., H. G. Heinze, R. Landgrap, K. W. Frey und K. Schwarz, Klin. Wschr. 44, 131 (1966). - 18. Lissitzky, S. und J. Brsmuth, Clin. chim. Acta (Amsterdam) 8, 269 (1964). - 19. GyertyáNFY, G. und J. FöLDES, diese Z. 5, 213 (1967). - 20. Oppenheimer, J. H. und M. I. Surks, J. Clin. Endocr. (Springfield) 21,456 (1964).
Dr. G. Gyertyánfy Budapest VIII, Ungarn Korányi S-u 2/a

\section{Enzymatic Determination of Glycerol and Glyceride-Glycerol in Plasma}

\author{
By J. Parijs, F. Barbier and P. Vermeire \\ From the University of Ghent Policlinic of Internal Medicine (Director: Prof. Dr. L. Remouchamps), Ghent (Belgium.)
}

(Eingegangen am 25. Oktober 1967)

Die von Wieland beschriebene Methode für die enzymatische Glycerinbestimmung im Plasma wurde im Enteiweißungsverfahren, der Reihenfolge der Zugabe der Reagenzien und der Zeit der Extinktionsmessung vereinfacht. Die Methode wurde für die Bestimmung von Glycerid-Glycerin im Plasma erweitert.

The method described by WIELAND for the enzymatic determination of glycerol in plasma was simplified in the deproteinization procedure, the sequence of adding the reagents and the time of reading the optical density. The method has been extended to the determination of glyceride-glycerol in plasma.

The enzymatic determination of glycerol has been elaborated by WIELAND $(1,2)$ and modified by SPINELLA and MAGER (3). The latter authors extended the method to the determination of glyceride-glycerol, Glycerol is phosphorylated to L(-)-glycerol-1-phosphate by ATP and $\left.G^{1}\right)(2,4)$. Under the influence of $\alpha-G D H$, glycerol-1-phosphate and NAD are converted into DOAP and NADH (5).

An alkaline medium with an excess of NAD is used to increase the equilibrium constant of this reaction.

Finally DOAP is trapped by hydrazine $(4,6)$.

The optimal $\mathrm{pH}$ for enzyme activity of glycerolkinase is 9,$8 ; \mathrm{Mg}^{++}$is needed as an activator (4).

We modified the method of WIELAND and SPINELLAMAGER as follows:

1. deproteinization by the Somogrr procedure (7).

2. except for GDH, all reagents were added to the filtrate as a mixture and not separately.

3. the increase of optical density was measured once, one hour after adding $\mathrm{GDH}$, and not at short intervals until a maximum value was attained.

1) Abbreviations: GK = glycerolkinase (ATP: glycerol phosphotranșferase EC 2.7.1.30); $\alpha-G D H=$ Glycerolphosphate dehydrogenase (L-glycerol-3-phosphate: NAD Oxidoreductase EC 1.1.1.8); DOAP = dihydroxy-acetone-phosphate.

2) Both reagents are to be titrated one against the other.

3) Bochringer Biochimica.

\section{Methods}

\section{Reagents}

Zinc sulphate $0.25 \mathrm{~N}^{2}$ )

Sodium hydroxide $0.25 \mathrm{~N}^{2}$ )

Buffer: glycine $0.2 \mathrm{M}$; hydrazine $1 \mathrm{M} ; \mathrm{Mg}^{++} 2 \cdot 10^{-3} \mathrm{M}, \mathrm{pH} 9.8$ Store at $4^{\circ} \mathrm{C}$ in a brown bottle and prepare fresh every 4 weeks. ATP 3 ) $0.05 \mathrm{M}$

Neutralised with $1 \mathrm{~N} \mathrm{NaOH}$, the solution is stable for several months.

$\mathrm{NAD}^{3}$ ) $0.02 \mathrm{M}$

Kept frozen in suitable portions

GK-suspension $\left.{ }^{3}\right) 1 \mathrm{mg} / \mathrm{ml}$

Kept at $4^{\circ} \mathrm{C}$.

GDH-suspension $\left.{ }^{3}\right) 10 \mathrm{mg} / \mathrm{ml}$

Kept at $4^{\circ} \mathrm{C}$.

Reagent-mixture

$5.50 \mathrm{~m} l$ buffer $; 0.20 \mathrm{~m} l$ ATP $; 0.20 \mathrm{~m} / \mathrm{NAD} ; 0.04 \mathrm{~m} /$ glycerolkinase. Incubated for 15 minutes at $37^{\circ} \mathrm{C}$ immediately before use. This mixture should be used within 2 hours after its preparation.

Bloor-mixture: ethanol/diethylether 3:1 (V/V)

Silicagel Merck $\mathrm{N}^{\circ} 7729$ - particle size below $0.08 \mathrm{~mm}$. Nonactivated.

Chloroform

Ethanol

Ethanolic $\mathrm{KOH}$-solution
a) stock solution (2\%)
b) work solution $(0.4 \%)$
Acetic acid (6\%)
Petroleum ether
Sulphuric acid $0.67 \mathrm{~N}$
Sodium hydroxide $0.67 \mathrm{~N}$ 